\title{
Prognostic and Predictive Value of Pretreatment Derived Neutrophil-to-Lymphocyte Ratio in Non-Small-Cell Lung Cancer Patients Treated with an Immune Checkpoint Inhibitor
}

\author{
John Kucharczyk ${ }^{1}$, Caitlin Sullivan², Jonathan Lu' ${ }^{3}$, Andrew Kolomensky², \\ Edward Peters ${ }^{4}$, Marc R. Matrana ${ }^{2,5^{*}}$
}

\begin{abstract}
${ }^{1}$ NYU Winthrop Hospital Internal Medicine Residency Program, NYU Winthrop Hospital, Mineola, NY, USA
${ }^{2}$ The University of Queensland School of Medicine, Ochsner Clinical School, New Orleans, LA, USA

${ }^{3}$ Ochsner Medical Oncology Fellowship Program, Ochsner Clinic Foundation, New Orleans, LA, USA

${ }^{4}$ Louisiana State University School of Public Health, New Orleans, LA, USA

${ }^{5}$ Ochsner Cancer Institute, Ochsner Clinic Foundation, New Orleans, LA, USA

Email: jkucharczyk@nyuwinthrop.org,v-csullivan@ochsner.org, jlu@ochsner.org, v-akolomensky@ochsner.org, epetel@lsuhsc.edu, ×mamatrana@ochsner.org
\end{abstract}

How to cite this paper: Kucharczyk, J., Sullivan, C., Lu, J., Kolomensky, A., Peters, E. and Matrana, M.R. (2018) Prognostic and Predictive Value of Pretreatment Derived Neutrophil-to-Lymphocyte Ratio in Non-Small-Cell Lung Cancer Patients Treated with an Immune Checkpoint Inhibitor. Journal of Cancer Therapy, 9, 23-34.

https://doi.org/10.4236/jct.2018.91004

Received: December 1, 2017

Accepted: January 22, 2018

Published: January 25, 2018

Copyright $\odot 2018$ by authors and Scientific Research Publishing Inc. This work is licensed under the Creative Commons Attribution International License (CC BY 4.0).

http://creativecommons.org/licenses/by/4.0/

c) (i) Open Access

\begin{abstract}
Background: Immune checkpoint inhibitors produce prolonged responses in select non-small cell lung cancer (NSCLC) patients, however the identification of patients most likely to benefit is difficult. Pretreatment derived neutrophil-to-lymphocyte ratio (dNLR) is an easily calculated marker available in routine clinical care that has shown prognostic value in many cancer treatment settings, but its association with survival in NSCLC patients treated with immune-checkpoint inhibitors is less understood. Patients and Methods: We retrospectively reviewed 72 NSCLC patients receiving either nivolumab or pembrolizumab between $3 / 1 / 15$ and 3/1/17 with a median follow-up time of 5.1 months. Patients were compared using Cox proportional hazards models to detect an association between pretreatment $\mathrm{dNLR}<3$ vs $\geq 3$ on overall survival (OS), progression-free survival (PFS) and overall response rate. Results: Median age was 65 (range: $41-86$ ), 65\% were male, $40 \%$ received $\geq 2$ prior systemic therapies and $14 \%$ had an Eastern Cooperative Oncology Group Performance Status (ECOG PS) $\geq 2$. Pretreatment dNLR $\geq 3$ was independently associated with shortened OS (median 3.6 vs 8.5 months; HR: 5.4; 95\% CI: 2.0 - 14.6; $\mathrm{p}=0.001$ ) and PFS (median 2.1 vs 3.4; HR: 2.3; 95\% CI: 1.1 - 4.8; $\mathrm{p}=0.027$ ). Conclusion: Pretreatment $\mathrm{dNLR} \geq 3$ was independently associated with inferior survival in NSCLC treated with immune checkpoint inhibitors in
\end{abstract}


routine practice. Prospective verification of this marker is warranted as it could serve as an inexpensive and widely-available marker for identifying NSCLC patients most likely to benefit from PD-1 inhibitors.

\section{Keywords}

Clinical Marker, PD-1 Inhibitor, Immunotherapy, Nivolumab, Pembrolizumab

\section{Introduction}

Immune checkpoint inhibitor therapies have been approved for multiple malignancies and have heralded a new era for cancer therapies. New data is rapidly materializing and clinical practices and protocols are changing quickly. Immune checkpoint inhibitors can prevent cancer cells from inhibiting the immune system's natural antineoplastic response by blocking inhibitory signaling pathways involving interactions between programmed death 1 (PD-1) receptors and its ligands (PD-L1 and PD-L2), thus enhancing the activity of T cells within the tumor microenvironment [1]. Targeted immunotherapy has been FDA-approved for non-small cell lung cancer (NSCLC) and has shifted treatment paradigms dramatically over the last few years.

Two programmed death 1 (PD-1) inhibitors, nivolumab and pembrolizumab, have particularly revolutionized the treatment of previously treated NSCLC. Nivolumab extended overall survival (OS) in phase III randomized controlled trials compared to second line docetaxel in both squamous-cell and non-squamous cell NSCLS [2] [3]. A second-line phase III study in patients with PD-L1 expression $\geq 50 \%$ demonstrated a survival advantage for pembrolizumab over docetaxel [4]. However, these registration trials featured low response rates $(18 \%-20 \%)$ and serious autoimmune complications that motivate a need to discover pretreatment clinical markers that identify patients most likely to benefit from a PD-1 inhibitor.

Most efforts to identify such a marker have focused on PD-L1 expression. This biomarker offers some prognostic information for patients beginning treatment with nivolumab or pembrolizumab, but cannot predict non-benefiting patients [5]. This is demonstrated by the results of the CheckMate 057 trial, in which although PD-L1 expression was shown to correlate with prolonged survival vs docetaxel in non-squamous cell NSCLS, durable responses were observed in patients with no PD-L1 expression [2].

As the predictive role of PD-L1 expression is nebulous, additional markers can help create a more robust system for identifying patients more likely to have a favorable clinical outcome. The pretreatment neutrophil-to-lymphocyte ratio (NLR) has been correlated with overall survival (OS) and progression free survival (PFS) in a wide range of malignancies, including melanoma patients treated with the immune-checkpoint inhibitor ipilimumab [6] [7] [8]. The derived NLR (dNLR), 
a formula involving exclusively white blood cell (WBC) count and Absolute Neutrophil Count (ANC), is an alternative to NLR when only WBC and ANC are available. The dNLR has been shown to have similar prognostic value to NLR in a large, multi-cancer study as well as a study of melanoma patients treated with ipilimumab, but its relationship to the survival of NSCLC patients treated with PD-1 inhibitors has not been evaluated to date [9] [10]. To that end, we evaluate the association between dNLR and survival of NSCLC patients treated with either nivolumab or pembrolizumab in an effort to describe a widely-available, low cost and effective marker to help identify patients most likely to benefit from this therapy.

\section{Methods}

\subsection{Patients}

We conducted a retrospective analysis of all NSCLC patients receiving either nivolumab or pembrolizumab within our health system between 3/1/15 and 3/1/17. Patients were excluded if they received less than two cycles of therapy. We included both PD-1 inhibitors to maximize sample size, although analysis on the nivolumab-only cohort was also done. Data collected from the electronic medical record include Eastern Cooperative Oncology Group Performance Status (ECOG PS), tumor histology, driver mutation status including EGFR, $A L K$ and $R O S$-1, PD-L1 expression, sites of metastases at initiation of PD-1 inhibitor, previous treatments and baseline complete blood count. Data on immune-related adverse events (irAEs), such as pneumonitis, dermatitis, hepatitis, arthritis, colitis and others, were graded using the National Cancer Institute's Common Terminology Criteria for Adverse Events version 4.0 (CTCAE v4.0) by a single investigator (J.K.) (Figure 1).

Pretreatment dNLR was calculated via the formula: $\mathrm{dNLR}=\mathrm{ANC} /(\mathrm{WBC}-\mathrm{ANC})$ [10]. OS was defined as the number of months between the initiation of therapy and either the death date or as the censored date of patient last contact. PFS was defined as the number of months between initiation of therapy and either progression by imaging, discontinuation of therapy, death (whichever occurred first), or censored as date of last imaging.

Treatment response was evaluated using either the Response Evaluation Criteria in Solid Tumors (RECIST) version 1.1 or the PET Response in Solid Tumors v 1.0 [11] [12]. Overall Response Rate (ORR) was defined as percentage of either Complete Response (CR) or Partial Response (PR) among all patients with response evaluation.

The Ochsner Medical Center institutional review board approved the project and informed consent was waived in this retrospective study.

\subsection{Statistical Analysis}

Demographic characteristics, safety and efficacy were described with descriptive statistics as either relative frequencies (percentages) or medians with ranges for quantitative variables. dNLR cut off values at intervals of 0.5 were compared 
(a)

OS total cohort

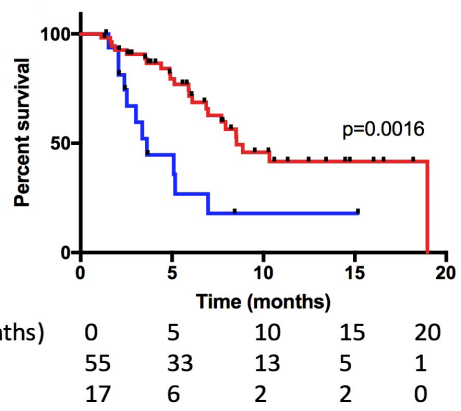

(c) OS in Nivolumab-treated patients

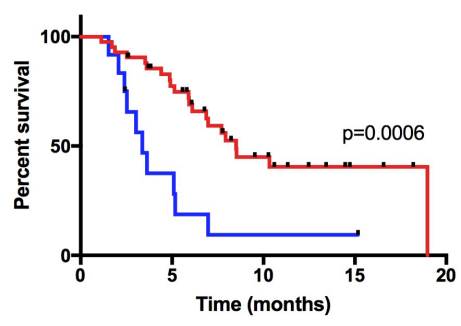

(b)

PFS total cohort

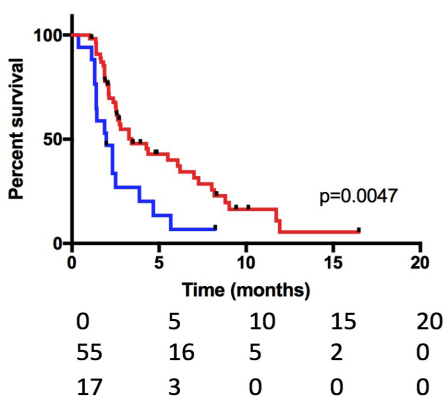

(d) PFS in Nivolumab-treated patients

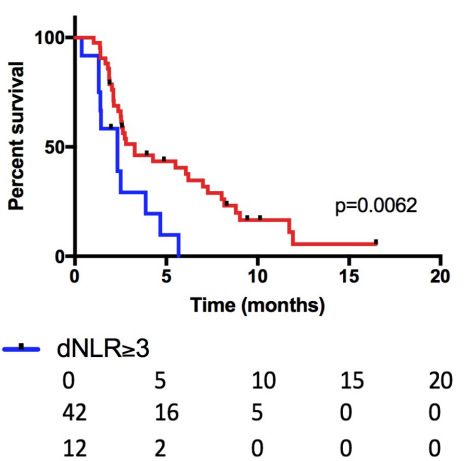

Figure 1. Survival of patients treated with both nivolumab and pembrolizumab and survival of patients only receiving nivolumab. (a), (b): Kaplan-Meier curves for overall survival (OS) and progression-free survival (PFS) in patients divided according to baseline derived Neutrophil-to-Lymphocyte (dNLR). (c), (d): Kaplan-Meier curves for OS and PFS in the nivolumab-only treated cohort. p-values represent log rank curve comparisons.

using Receiver Operator Curves (ROC) with analysis of the Area Under the Curve (AUC) using OS as an end-point. Kaplan-Meier analysis was used to estimate OS and PFS with differences in curves analyzed with log-rank test. Cox proportional hazard regression models were used to estimate hazard ratios (HR) and P-values for the relationship between dNLR and OS and PFS. The final multivariate model included age, sex, race, ECOG PS, smoking history, squamous histology, number of prior medical therapies and site of metastatic disease. Exploratory analysis for the contribution of ANC and ALC was conducted both by dividing the cohort with previously established cutoffs (750 for ANC; 1000 for ANC) and by dividing the cohort into quartiles based on either their ANC or ALC [9] [13]. These univariate analyses were also compared with the Kaplan-Meier method and log-rank test.

For all analysis, $\mathrm{p}<0.05$ was considered significant. The statistical analysis was performed in Stata version 14.2 (Stata Corp., College Station, Texas).

\section{Results}

\subsection{Patient Characteristics}

Between March 2015 and April 2017, 72 NSCLC patients were treated with at 
least 2 cycles of a PD-1 inhibitor at our institution. Among them, 54 (75\%) received nivolumab, and $18(25 \%)$ received pembrolizumab. Median follow up from the initiation of therapy was 5.1 months, $34(47.2 \%)$ of patients died as of April 2017. Patient characteristics are listed in Table 1 . The median age at initiation of treatment was 65 (Range 41 - 86), 47 (65\%) patients were male, 37 (51\%) were Caucasian while 30 (41\%) were African-American. Eighty-six percent of patients had an ECOG PS of 0 - 1 and 51 (74\%) had a history of heavy smoking. $20(28 \%)$ had bone metastases at initiation of therapy, $13(18 \%)$ had liver and 11 (15\%) had brain. Half of the cohort received a PD-1 inhibitor in the second line setting.

Among the $57(79.1 \%)$ patients with non-squamous histology, 47 (82\%) had molecular testing for $E G F R$ mutation, 42 (72\%) for $A L K$ translocation and 32 (56\%) for $R O S-1$ translocation. Of the 15 (21\%) patients with squamous cell histology, 3 (20\%) had EGFR mutation testing and 2 (13\%) had $A L K$ translocation testing.

\subsection{Survival}

The ROC curve established the optimal dNLR cutoff by comparing the sensitivity and specificity of different thresholds. The optimal cutoff was determined to be 3; AUC was 0.722 ( $\mathrm{p}<0.006$ ). Median survival in the entire cohort was 7.9 months. Patients with $\mathrm{dNLR}<3$ had superior $\mathrm{OS}$ in the unadjusted analysis (median 8.5 vs 3.6 months; HR 2.35, 95\% CI 1.15 - 4.82; p = 0.002). In multivariate analysis, elevated ECOG PS $(\geq 2)$, the presence of liver metastases, squamous cell histology and $\mathrm{dNLR} \geq 3$ were significantly associated with shortened OS, baseline dNLR $<3$ was associated with prolonged OS (HR: 5.4; 95\% CI: 2.0 14.6; $\mathrm{p}=0.001$ ) (Table 2). In the subset of patients receiving nivolumab, the same multivariate analysis revealed a similar association (HR: 7.9; 95\% CI: 2.8 22.5; $\mathrm{p}<0.001)$.

Median PFS was 2.7 months, 54 (75\%) subjects had progressed at the time of analysis. $\mathrm{dNLR}<3$ was associated with superior PFS on crude analysis (3.4 vs 2.0 months; HR 1.75, 95\% CI 0.96 - 3.17; $\mathrm{p}=0.005)$. Baseline dNLR and treatment were the only variables associated with PFS in multivariate analysis. In the final multivariate model for PFS, elevated dNLR remained independently associated with prolonged PFS (HR: 2.3; 95\% CI: 1.1 - 4.8; $\mathrm{p}=0.027$ ). In a multivariate analysis of the nivolumab-only cohort, $\mathrm{dNLR} \geq 3$ was also independently associated with PFS (HR: 2.8; 95\% CI: 1.2 - 46.4; $\mathrm{p}=0.018$ ).

\subsection{Response}

Treatment responses were available for 63 patients. The ORR was $20.6 \%$, with one patient experiencing complete response (CR), 12 partial response (PR), 28 stable disease (SD) and 22 progressive disease (PD) as best response. dNLR was not associated with response to treatment in multivariate analysis (OR: 5.45 ; 95\% CI: $0.34-88.65 ; \mathrm{p}=0.233$ ), nor were the other variables included in the 
Table 1. Patient characteristics.

\begin{tabular}{|c|c|}
\hline Characteristic & $\mathrm{N}(\%)$ \\
\hline \multicolumn{2}{|l|}{ Age } \\
\hline Median & 65 \\
\hline Range & $41-86$ \\
\hline$<75$ & $60(83.3)$ \\
\hline Male & $47(65.3)$ \\
\hline \multicolumn{2}{|l|}{ Race } \\
\hline White & $37(51.4)$ \\
\hline Black & $30(41.7)$ \\
\hline Other & $3(4.2)$ \\
\hline \multicolumn{2}{|l|}{ ECOG PS ${ }^{\mathrm{a}}$} \\
\hline 0 & $26(36.1)$ \\
\hline 1 & $36(50)$ \\
\hline 2 & $10(13.9)$ \\
\hline 3 & $0(0)$ \\
\hline \multicolumn{2}{|l|}{ Smoking history ${ }^{\mathrm{b}}$} \\
\hline Light/never & $18(25)$ \\
\hline Heavy & $51(70.8)$ \\
\hline \multicolumn{2}{|l|}{ Histology } \\
\hline Non-squamous & $57(79.2)$ \\
\hline Squamous & $15(20.8)$ \\
\hline \multicolumn{2}{|c|}{ Targetable driver mutation $^{c}$} \\
\hline EGFR & $9(12.5)$ \\
\hline ALK & 0 \\
\hline ROS-1 & 0 \\
\hline \multicolumn{2}{|c|}{ Site of metastases at initiation of therapy } \\
\hline Bone & $20(27.8)$ \\
\hline Liver & $13(18.1)$ \\
\hline Brain & $11(15.3)$ \\
\hline \multicolumn{2}{|l|}{ Line of therapy } \\
\hline 2 & $36(50)$ \\
\hline 3 & $20(27.8)$ \\
\hline $4+$ & $9(12.5)$ \\
\hline \multicolumn{2}{|l|}{ Therapy } \\
\hline Nivolumab & $54(75)$ \\
\hline Pembrolizumab & $18(25)$ \\
\hline \multicolumn{2}{|l|}{ dNLR } \\
\hline$<3$ & $55(76.4)$ \\
\hline$\geq 3$ & $17(23.6)$ \\
\hline
\end{tabular}

${ }^{a}$ ECOG PS: Eastern Cooperative Oncology Group Performance Status; ${ }^{b} H e a v y$ ( $\geq 10$ pack-years), light/never ( $<10$ pack-years); 'EGFR: Epidermal Growth Factor Receptor; ALK: Anaplastic Lymphoma Kinase; ROS1: ROS1 oncogene. 
Table 2. Multivariate analyses.

\begin{tabular}{ccccccc}
\hline & \multicolumn{3}{c}{ Overall Survival } & \multicolumn{2}{c}{ Progression-Free Survival } \\
\hline Parameter & $H \boldsymbol{R}$ & $\boldsymbol{P}$ & $\mathbf{9 5 \%} \boldsymbol{C I}$ & HR & $\boldsymbol{P}$ & $95 \% \boldsymbol{C I}$ \\
\hline dNLR $\geq 3$ & 5.41 & 0.001 & $2.01-14.56$ & 2.29 & 0.027 & $1.10-4.79$ \\
Age & 0.96 & 0.113 & $0.92-1.01$ & 0.99 & 0.496 & $0.96-1.02$ \\
Sex & 0.94 & 0.879 & $0.40-2.18$ & 0.92 & 0.809 & $0.46-1.84$ \\
Race & 0.56 & 0.222 & $0.22-1.42$ & 0.89 & 0.729 & $0.47-1.68$ \\
Smoking & 0.38 & 0.002 & $0.20-0.70$ & 0.73 & 0.195 & $0.46-1.17$ \\
Treatment line & 1.41 & 0.115 & $0.92-2.16$ & 1.45 & 0.024 & $1.05-1.99$ \\
ECOG PS & 1.79 & 0.031 & $1.06-3.05$ & 1.50 & 0.062 & $0.98-2.30$ \\
Liver metastases & 4.46 & 0.005 & $1.58-12.61$ & 1.76 & 0.162 & $0.80-3.90$ \\
Brain metastases & 0.57 & 0.378 & $0.17-1.97$ & 0.89 & 0.809 & $0.36-2.21$ \\
Bone metastases & 2.10 & 0.088 & $0.90-4.93$ & 1.23 & 0.55 & $0.62-2.46$ \\
Squamous histology & 5.58 & 0.001 & $2.06-15.14$ & 1.74 & 0.106 & $0.89-3.40$ \\
\hline
\end{tabular}

final logistic regression model (age, sex, race, smoking status, ECOG PS, treatment line, liver, brain, bone metastases or histology).

\subsection{Exploratory Analysis of ANC and ALC}

To evaluate the contribution of ANC and ALC to the survival benefits of low dNLR, we evaluated the effect of each variable on survival by dividing the cohort into quartiles. Of note, only 41 patients had ALC data available. ANC quartiles were $1.50-3.30(\mathrm{n}=18), 3.30-4.72(\mathrm{n}=18), 4.76-6.60(\mathrm{n}=18)$ and 6.65 $16.42(\mathrm{n}=18)$; ALC quartiles were $0.45-0.89(\mathrm{n}=11), .91-1.27(\mathrm{n}=10), 1.31$ $1.67(\mathrm{n}=10)$ and $1.67-2.93(\mathrm{n}=10)$. As shown in Figure 2, there was no significant difference between quartiles divided by ANC (log-rank $\mathrm{p}=0.88$ ) but there was for ALC (log-rank $\mathrm{p}=0.0258)$. When dividing the curves by previously determined cutoff points for ANC (7.5) and ALC (1.0), there was no difference in survival for the patients separated by ANC (log-rank $\mathrm{p}=0.24)$ while there was for those separated by ALC (log-rank $\mathrm{p}=0.0024)$.

\subsection{Immune Related Adverse Events}

The frequency and grade of the irAEs observed are summarized in Table 3. Overall, 16 (22\%) of patients experienced a total of $19 \mathrm{irAEs,} \mathrm{of} \mathrm{which} 15$ (79\%) were graded 1/2. irAEs were experienced after a median of 7 cycles.

\section{Discussion}

Immune checkpoint inhibitors have radically altered the therapeutic compendia for metastatic NSCLC. Indeed, nivolumab and pembrolizumab have been shown to prolong OS with favorable toxicity profiles [2] [3] [4]. But, there remains a great need for biomarkers to stratify patients most likely to benefit from these 
(a)

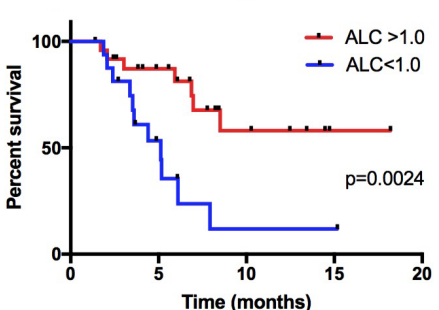

Time (months)

ALC $\geq 1.0$

ALC $<1.0$

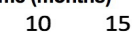

$\begin{array}{lllll}16 & 7 & 10 & 15 & 20\end{array}$

$\begin{array}{llllll}25 & 17 & 7 & 2 & 0 & \text { ALC } \geq 7.5\end{array}$

(b)

OS by ANC

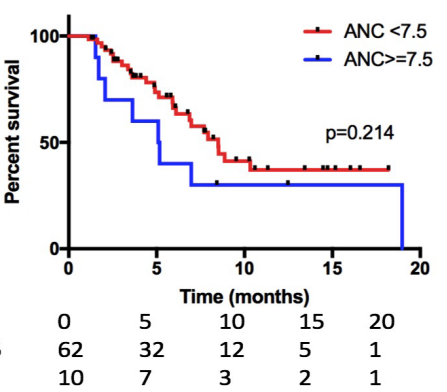

(d) OS by ANC quartiles

(c) Os by ALC quartiles
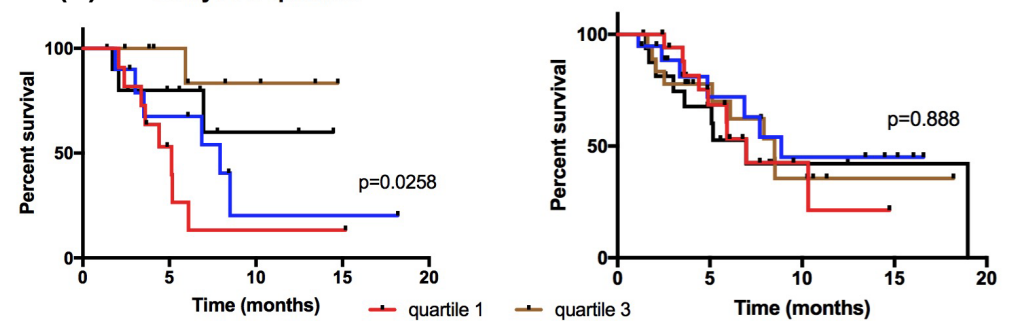

$\begin{array}{lllllllllll}\text { Time (months) } & 0 & 5 & 10 & 15 & 20 & 0 & 5 & 10 & 15 & 20 \\ \text { Quartile 1 } & 11 & 5 & 2 & 2 & 0 & 18 & 11 & 3 & 1 & 0 \\ \text { Quartile 2 } & 10 & 7 & 2 & 2 & 0 & 18 & 9 & 6 & 4 & 0 \\ \text { Quartile 3 } & 10 & 7 & 4 & 1 & 0 & 18 & 11 & 5 & 2 & 0 \\ \text { Quartile 4 } & 10 & 7 & 3 & 1 & 0 & 18 & 10 & 3 & 2 & 0\end{array}$

Figure 2. Exploratory analysis of effect on ALC and ANC to overall survival (OS). (a) OS in patients divided into two groups by Absolute Lymphocyte Count (ALC); (b) OS of patients divided into two groups by Absolute Neutrophil Count (ANC); (c) OS of patients divided by ALC quartiles; (d) OS divided by ANC quartiles. p values represent log rank curve comparisons.

Table 3. Immune related adverse events.

\begin{tabular}{ccc}
\hline \multirow{2}{*}{ Adverse event } & \multicolumn{2}{c}{ N (\%) } \\
\cline { 2 - 3 } & Any Grade & Grade 3/4 \\
\hline Total events & 19 & 4 \\
Number of patients experiencing event & $16(22.2)$ & $4(5.6)$ \\
Dermatitis & $5(6.9)$ & \\
Hypothyroidism & $4(5.5)$ & $2(2.8)$ \\
Pneumonitis & $4(5.5)$ & \\
Mucositis & $2 .(2.8)$ & $1(1.4)$ \\
Transaminitis & $1(1.4)$ & $1(1.4)$ \\
Hypophysitis & $1(1.4)$ & \\
Myalgia & $1(1.4)$ & \\
Colitis & $1(1.4)$ & \\
\hline
\end{tabular}

therapies. In this 72 patient retrospective analysis of NSCLC patients treated with immunotherapy, pretreatment $\mathrm{dNLR}<3$ was associated with prolonged 
survival and PFS. Due to the widespread availability of dNLR in routine clinical practice, we suggest that dNLR should continue to be validated as a potential marker for identifying patients most likely to respond to immune checkpoint inhibitors.

Neutrophilia with relative lymphocytopenia has been shown to portend a worse prognosis in a multitude of solid tumors [8]. This is thought to be a result of nonspecific inflammation in the tumor microenvironment delivering bioactive factors that facilitate proliferation, angiogenesis, invasion and limit cell death [14]. In the setting of immune checkpoint-inhibitors, which block negative regulators of lymphocyte function, it is plausible that lymphocytosis would enhance the effect of these therapies. These hypotheses have been validated in multiple studies on ipilimumab-treated melanoma, in which elevated NLR was associated with decreased survival [6] [15] [16]. Additionally, a retrospective review of ipilimumab-treated melanoma patients similarly determined dNLR of 3 to be the ideal cutoff point and associated elevated dNLR with shortened survival [9].

Recently, Bagley et al found NLR $>5$ to be associated with inferior OS and PFS in nivolumab-treated NSCLC patients [17]. Our results are largely confirmatory, with the nuance of using dNLR instead of NLR due to the blood counts available at our institution. Although dNLR has been validated as roughly equivalent to NLR, we hope the present study provides additional confidence to providers who only have access to dNLR in their clinical practice [10].

Our findings did differ, however, in the relative contributions of ANC and ALC. We found no difference in survival in groups separated by ANC quartiles, while there was marked separation in survival curves when patients were separated by both ALC quartiles or divided by a previously established ALC cutoff [17] [18]. This discrepancy underscores the need to validate the application of these markers prospectively and in a larger cohort, as well as the advantage of a marker that captures both neutropenia and lymphocytosis. Pre- and post-treatment ALC has been associated with OS and PFS in ipilimumab-treated melanoma, but to date no reports have made that association in NSCLC [13] [19].

Our study population had inferior OS and PFS to that of the NSCLC registration trials [2] [3] [4]. This can be explained by our real-world population many who would have not qualified for a trial. Our patients were older (65 vs 61 - 63), received more previous therapies ( $40 \%$ receiving $\geq 2$ previous lines vs $0 \%-12 \%$ ) and had an inferior ECOG PS (14\% $\geq 2$ vs $0 \%-1 \%)$. Our multivariate analysis reveals that the inferior OS and PFS in our cohort is largely driven by inferior ECOG PS, which had a significant effect on OS, while age and line of therapy did not. The differences in the baseline characteristics of our cohort from those of the registration trials underscores the importance of describing real-world clinical experience with immune checkpoint inhibitors in NSCLC, on which very little has been reported. The rates of grade $3 / 4$ irAEs were similar ( $6 \%$ vs $5 \%$ ) to those of the registration trials, with hypothyroidism and pneumonitis being the most commonly observed [4]. 
This study has multiple limitations. We included two drugs, albeit with identical mechanisms, as it was felt that maximizing sample size yielded more powerful results, particularly in the exploratory analysis. To address this, analysis on the nivolumab-only cohort was conducted in parallel for our central conclusions. Additionally, we conducted formal RECIST measurements for our treatment response analysis, which would not account for pseudoprogression. Due to the predominant use of traditional RECIST criteria over immune-related response criteria in NSCLC PD-1 inhibitor research, and low incidence of pseudoprogression described in real-world populations, it was decided that RECIST was an appropriate, formalized means to track progression and response in this study [17] [20]. Furthermore, data on driver mutations, PD-L1 expression and Lactate Dehydrogenase (LDH) was not available to an extent that allowed its inclusion in the multivariate analyses. Finally, the present study is retrospective in nature and potentially vulnerable to biases and confounding inherent in this type of research. We attempted to identify and address confounding factors by building multivariate models.

\section{Conclusion}

Pretreatment dNLR $<3$ was independently associated with prolonged OS and PFS in patients receiving a PD-L1 inhibitor for NSCLC. Given the possible ability of NLR to identify patients most likely to benefit from these therapies, we encourage prospective, adequately powered studies to validate our findings.

\section{Clinical Practice Points}

- Not all NSCLC patients derive benefit from immune checkpoint inhibitors, and so a pretreatment clinical marker could be useful for stratifying patients most likely to benefit.

- $\mathrm{dNLR}$ is an inexpensive and widely-available marker that is easily calculated from a complete blood count.

- To date, the association of NLR and survival of patients receiving an immune checkpoint inhibitor has largely only been evaluated in melanoma patients.

- Our study consisted of 72 NSCLC patients, 75\% received nivolumab and 25\% received pembrolizumab. Patients were dichotomized according to pretreatment $\mathrm{dNLR}<3$ vs $\geq 3$.

- $\mathrm{dNLR} \geq 3$ was independently associated with shortened OS (median 3.6 vs 8.5 months) and PFS (median 2.1 vs 3.4) in cox proportional hazards models.

- dNLR should be prospectively verified as a potential marker to identify NSCLC patients most likely to benefit from PD-1 inhibitors.

\section{Funding}

This research did not receive any specific grant from funding agencies in the public, commercial, or not-for-profit sectors. 


\section{References}

[1] Pardoll, D.M. (2012) The Blockade of Immune Checkpoints in Cancer Immunotherapy. Nature Reviews Cancer, 12, 252-264. https://doi.org/10.1038/nrc3239

[2] Borghaei, H., Paz-Ares, L., Horn, L., et al. (2015) Nivolumab versus Docetaxel in Advanced Nonsquamous Non-Small-Cell Lung Cancer. New England Journal of Medicine, 373, 1627-1639. https://doi.org/10.1056/NEJMoa1507643

[3] Brahmer, J., Reckamp, K.L., Baas, P., et al. (2015) Nivolumab versus Docetaxel in Advanced Squamous-Cell Non-Small-Cell Lung Cancer. New England Journal of Medicine, 373, 123-135. https://doi.org/10.1056/NEJMoa1504627

[4] Herbst, R.S., Baas, P., Kim, D.W., et al. (2016) Pembrolizumab versus Docetaxel for Previously Treated, PD-L1-Positive, Advanced Non-Small-Cell Lung Cancer (KEYNOTE-010): A Randomised Controlled Trial. Lancet, 387, 1540-1550. https://doi.org/10.1016/S0140-6736(15)01281-7

[5] Grigg, C. and Rizvi, N.A. (2016) PD-L1 Biomarker Testing for Non-Small Cell Lung Cancer: Truth or Fiction? Journal for Immunotherapy of Cancer, 4, 48. https://doi.org/10.1186/s40425-016-0153-x

[6] Ferrucci, P.F., Gandini, S., Battaglia, A., et al. (2015) Baseline Neutrophil-to-Lymphocyte Ratio Is Associated with Outcome of Ipilimumab-Treated Metastatic Melanoma Patients. British Journal of Cancer, 112, 1904-1910.

https://doi.org/10.1038/bjc.2015.180

[7] Di Giacomo, A.M., Ascierto, P.A., Queirolo, P., et al. (2015) Three-Year Follow-Up of Advanced Melanoma Patients Who Received Ipilimumab Plus Fotemustine in the Italian Network for Tumor Biotherapy (NIBIT)-M1 Phase II Study. Annals of Oncology, 26, 798-803. https://doi.org/10.1093/annonc/mdu577

[8] Guthrie, G.J., Charles, K.A., Roxburgh, C.S., Horgan, P.G., McMillan, D.C. and Clarke, S.J. (2013) The Systemic Inflammation-Based Neutrophil-Lymphocyte Ratio: Experience in Patients with Cancer. Critical Reviews in Oncology/Hematology, 88, 218-230. https://doi.org/10.1016/j.critrevonc.2013.03.010

[9] Ferrucci, P.F., Ascierto, P.A., Pigozzo, J., et al. (2017) Baseline Neutrophils and Derived Neutrophil-to-Lymphocyte Ratio: Prognostic Relevance in Metastatic Melanoma Patients Receiving Ipilimumab. Annals of Oncology, 27, 732-738. https://doi.org/10.1093/annonc/mdw016

[10] Proctor, M.J., McMillan, D.C., Morrison, D.S., Fletcher, C.D., Horgan, P.G. and Clarke, S.J. (2012) A Derived Neutrophil to Lymphocyte Ratio Predicts Survival in Patients with Cancer. British Journal of Cancer, 107, 695-699.

https://doi.org/10.1038/bjc.2012.292

[11] Eisenhauer, E.A., Therasse, P., Bogaerts, J., et al. (2009) New Response Evaluation Criteria in Solid Tumours: Revised RECIST Guideline (Version 1.1). European Journal of Cancer, 45, 228-247. https://doi.org/10.1016/j.ejca.2008.10.026

[12] Wahl, R.L., Jacene, H., Kasamon, Y. and Lodge, M.A. (2009) From RECIST to PERCIST: Evolving Considerations for PET Response Criteria in Solid Tumors. Journal of Nuclear Medicine, 50, 122S-150S. https://doi.org/10.2967/jnumed.108.057307

[13] Berrocal, A., Arance, A., Lopez Martin, J.A., et al. (2014) Ipilimumab for Advanced Melanoma: Experience from the Spanish Expanded Access Program. Melanoma Research, 24, 577-583. https://doi.org/10.1097/CMR.0000000000000108

[14] Hanahan, D. and Weinberg, R.A. (2011) Hallmarks of Cancer: The Next Generation. Cell, 144, 646-674. https://doi.org/10.1016/j.cell.2011.02.013 
[15] Cassidy, M.R., Wolchok, R.E., Zheng, J., et al. (2017) Neutrophil to Lymphocyte Ratio Is Associated with Outcome during Ipilimumab Treatment. EBioMedicine, 18, 56-61. https://doi.org/10.1016/j.ebiom.2017.03.029

[16] Khoja, L., Atenafu, E.G., Templeton, A., et al. (2016) The Full Blood Count as a Biomarker of Outcome and Toxicity in Ipilimumab-Treated Cutaneous Metastatic Melanoma. Cancer Medicine, 5, 2792-2799. https://doi.org/10.1002/cam4.878

[17] Bagley, S.J., Kothari, S., Aggarwal, C., et al. (2017) Pretreatment Neutrophil-to-Lymphocyte Ratio as a Marker of Outcomes in Nivolumab-Treated Patients with Advanced Non-Small-Cell Lung Cancer. Lung Cancer, 106, 1-7. https://doi.org/10.1016/j.lungcan.2017.01.013

[18] Ku, G.Y., Yuan, J., Page, D.B., et al. (2010) Single-Institution Experience with Ipilimumab in Advanced Melanoma Patients in the Compassionate Use Setting: Lymphocyte Count after 2 Doses Correlates with Survival. Cancer, 116, 1767-1775. https://doi.org/10.1002/cncr.24951

[19] Kelderman, S., Heemskerk, B., van Tinteren, H., et al. (2014) Lactate Dehydrogenase as a Selection Criterion for Ipilimumab Treatment in Metastatic Melanoma. Cancer Immunology, Immunotherapy, 63, 449-458.

[20] Nishino, M., Ramaiya, N.H., Chambers, E.S., et al. (2016) Immune-Related Response Assessment during PD-1 Inhibitor Therapy in Advanced Non-Small-Cell Lung Cancer Patients. Journal for Immunotherapy of Cancer, 4, 84.

https://doi.org/10.1186/s40425-016-0193-2 